\title{
In Situ and Ex Situ Energy-Filtered Transmission Electron Microscopy Studies on the Nanomorpholgy Evolution of Organic Bulk Heterojunction Solar Cells
}

\author{
Christina Harrei $ß^{1 *}$, Stefan Langner ${ }^{2}$, Marvin Berlinghof ${ }^{3}$, Stefanie Rechberger ${ }^{1}$, Johannes Will ${ }^{1}$, Tobias \\ Unruh $^{3}$, Christoph J. Brabec ${ }^{2}$, Erdmann Spiecker ${ }^{*}$ \\ 1. Institute of Micro- and Nanostructure Research (IMN) \& Center for Nanoanalysis and Electron \\ Microscopy (CENEM), University of Erlangen-Nuremberg, Germany \\ 2. Institute of Materials for Electronics and Energy Technology (i-MEET), University of Erlangen- \\ Nuremberg, Germany \\ 3. Institute for Crystallography and Structural Physics, University of Erlangen-Nuremberg, Germany \\ * Corresponding authors: christina.harreiss@fau.de, erdmann.spiecker@fau.de
}

The power conversion efficiency (PCE) of solution-processed bulk heterojunction (BHJ) solar cells has seen a dramatic increase in the last decades due to the introduction of new donor and acceptor materials, as well as the development of new processing conditions [1][2]. By mixing an electron donor with an electron acceptor a certain nanomorphology of the two phases is evolving depending on the composite materials, the applied solvent and the post-processing conditions like, e.g., solvent vapor (SVA) or thermal annealing (TA) (Figure 1). Since the nanoscale morphology of organic BHJ solar cells determines the charge separation at the interfaces and the subsequent electron and hole transport to the respective electrode, it decisively influences the device performance. Therefore, understanding and controlling the nanostructure evolution is a necessity to further improve the device performance.

Electron energy-loss spectroscopy (EELS) and energy-filtered transmission electron microscopy (EFTEM) are powerful techniques for studying the material distribution and nanomorphology of donor and acceptor phases since conventional TEM imaging techniques are mostly not sufficient to visualize and identify the small molecule and fullerene component. Due to their similar densities and complex crystallinity, conventional TEM delivers rather uniform contrast where no significant structures can be identified. In contrast, by using either ionization edge or plasmon loss electrons in EFTEM pronounced contrast can be generated enabling reliable discrimination of the two phases.

Here we report on a high-efficiency photovoltaic system composed of a small molecule 2,2'[(3,3"',3"',,4'-tetraoctyl[2,2':5',2":5",2"':5"',2"'--quinquethiophene]-5,5'"'-diyl)bis[(Z)-methylidyne(3-ethyl4-oxo-5,2-thiazolidinediylidene)]]bis-propanedinitrile (DRCN5T) electron donor and [6,6]-phenyl C71 butyric acid methyl ester $\left(\mathrm{PC}_{71} \mathrm{BM}\right)$ as electron acceptor. We investigated the influence of three different solvent atmospheres on the nanomorphology, crystallinity and efficiency of our organic solar cell system. The three solvents show either a high solubility towards the donor (THF) or towards the acceptor $\left(\mathrm{CS}_{2}\right)$ or show a high solubility for both materials $\left(\mathrm{CHCl}_{3}\right)$. We studied the nanomorpholgy evolution as a function of the solvent vapor atmosphere and the annealing time using EFTEM imaging techniques (Figure 2). Thereby, we figured out that the choice of the solvent for SVA has a high impact on the evolving nanomorpholgy and the degree and speed of phase separation and crystal growth. The crystallinity of the annealed and as-casted samples was investigated by energy-filtered electron diffraction (EF-ED) measurements where an energy selecting slit was positioned around the zero-loss peak in order to enhance the contrast of elastic scattering and Bragg diffraction. Furthermore, we have shown that grazing-incidence wide-angle X-ray scattering (GIWAXS) can be used as a complementary technique and provides results in good agreement with EF-ED. The nanomorpholgy was then further correlated to the device performance. By that, an in-depth understanding of the relationship between post-processing, morphology, crystallinity and device performance could be gained. 
Currently we are performing in situ thermal annealing experiments in the TEM enabling the simultaneous observation of the nanoscale phase separation and the formation of an interpenetrating network of donor and acceptor phases as a function of the annealing time and temperature. This gives us new insights into the kinetics of BHJ film formation.

\section{References:}

[1] J Min et al., J. Mater. Chem. A 5 (2017), p. 18101-18110.

[2] J Min et al., Nano Energy 28 (2016), p. 241-249.

[3] The authors acknowledge the financial support of the DFG within the framework of the collaborative research center SFB 953 "Synthethic Carbon Allotropes".
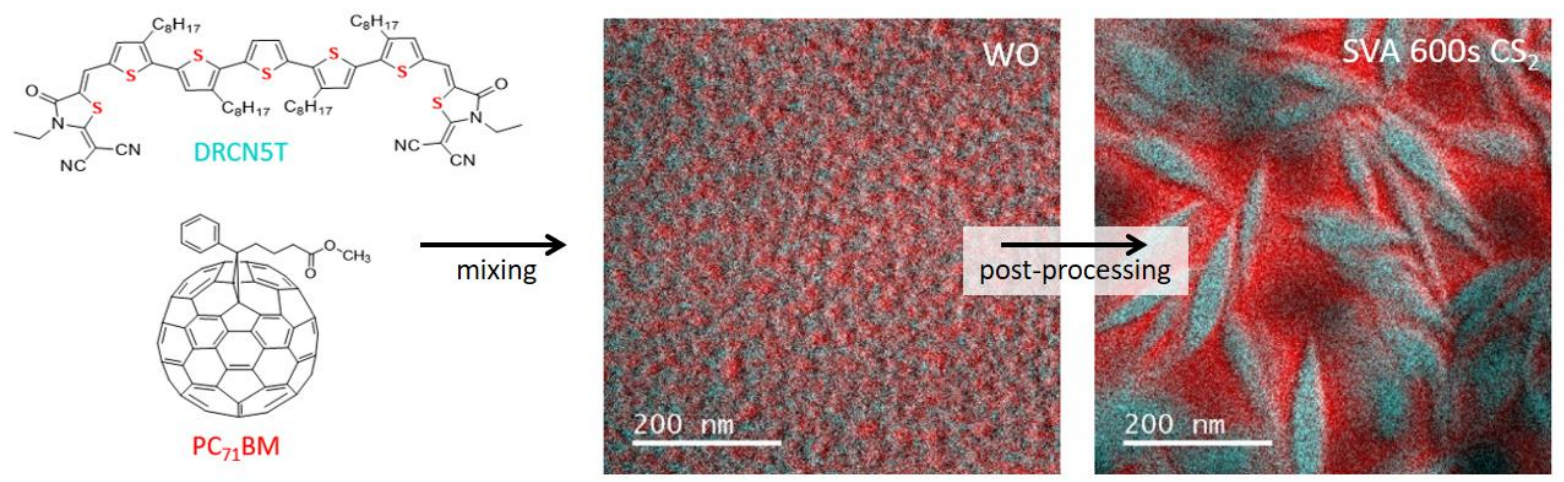

Figure 1. Scheme of the solution-processing of organic bulk heterojunction blends and their nanomorpholgy evolution due to post-processing treatments (DRCN5T: cyan; PC 71 BM: red).
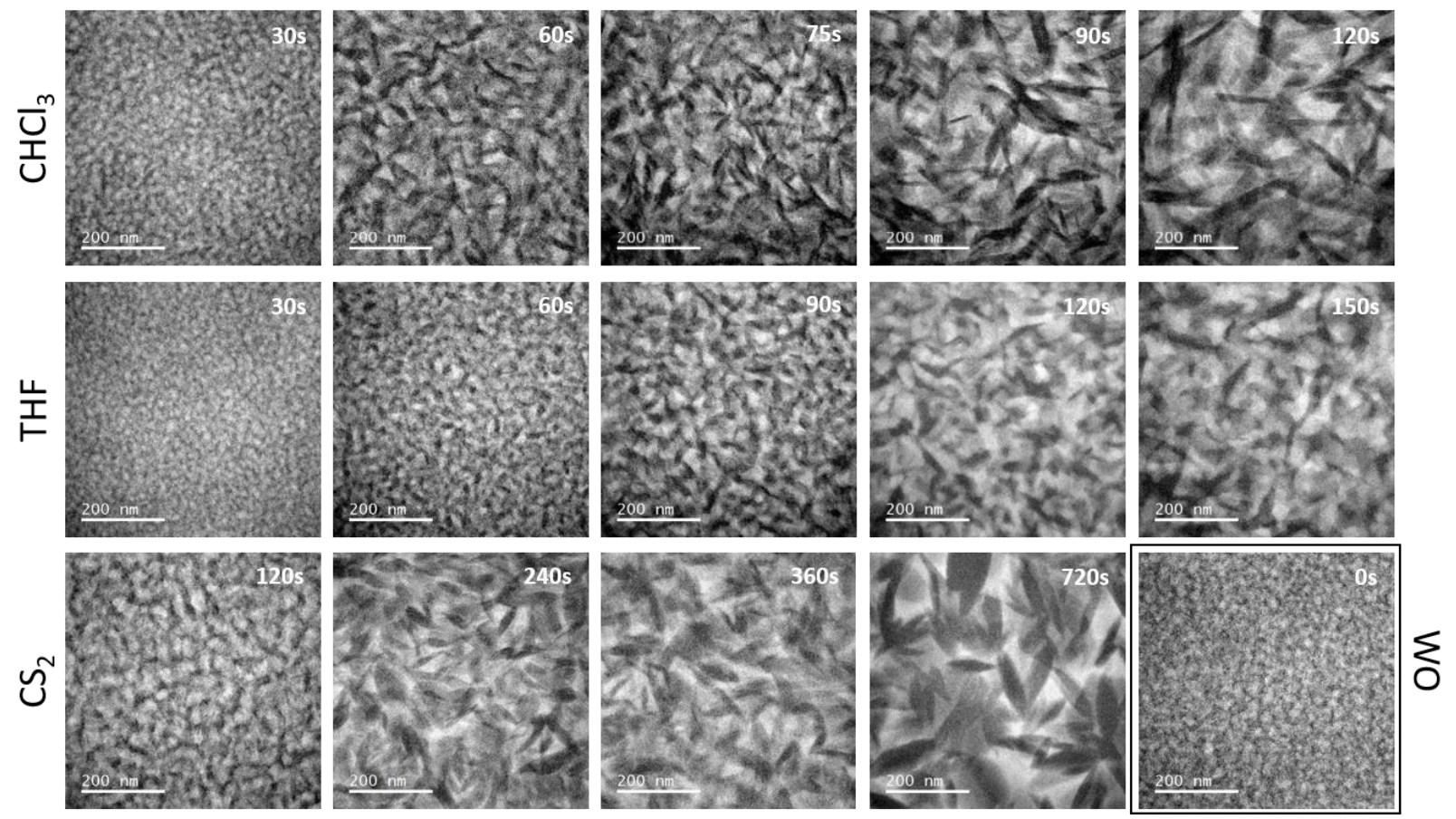

Figure 2. EFTEM elemental maps of carbon for different solvent vapor atmospheres and various annealing times (WO: reference without post-treatment). 